\title{
The validity and reliability of four-meter gait speed test for stable interstitial lung disease patients: the prospective study
}

\author{
Ryosuke Hirabayashi" ${ }^{1 \#}$, Yusuke Takahashi" ${ }^{2 \#}$ Kazuma Nagata ${ }^{1 \#}$, Takeshi Morimoto ${ }^{3,4}$, Kyosuke Wakata ${ }^{5}$, \\ Atsushi Nakagawa ${ }^{1}$, Ryo Tachikawa ${ }^{1}$, Kojiro Otsuka ${ }^{6}$, Keisuke Tomii ${ }^{1}$ \\ ${ }^{1}$ Department of Respiratory Medicine, Kobe City Medical Center General Hospital, 2-1-1 Minatojima-minamimachi, Chuo-ku, Kobe, Hyogo, 650- \\ 0047, Japan; ${ }^{2}$ Department of Rehabilitation, Kaetsu Hospital, 1459-1, Higashi-kanazawa, Akiba-ku, Niigata, Niigata, 956-0854, Japan; ${ }^{3}$ Department \\ of Clinical Reserch Center, Kobe City Medical Center General Hospital, 2-1-1 Minatojima-minamimachi, Chuo-ku, Kobe, Hyogo, 650-0047, \\ Japan; ${ }^{4}$ Department of Clinical Epidemiology, Hyogo College of Medicine, 1-1 Mukogawa-cho, Nishinomiya, Hyogo, Japan; ${ }^{5}$ Department \\ of Rehabilitation, Kobe City Medical Center General Hospital, 2-1-1 Minatojima-minamimachi, Chuo-ku, Kobe, Hyogo, 650-0047, Japan; \\ ${ }^{6}$ Department of Respiratory medicine, Shinko Hospital, 1-4-47 Wakihama-cho, Chuo-ku, Kobe, Hyogo, 651-0072, Japan \\ Contributions: (I) Conception and design: R Hirabayashi, Y Takahashi, K Nagata; (II) Administrative support: K Nagata; (III) Provision of \\ study materials or patients: R Hirabayashi, K Nagata; (IV)Collection and assembly of data: R Hirabayashi, Y Takahashi; (V) Data analysis and \\ interpretation: R Hirabayashi, K Nagata; (VI) Manuscript writing: All authors; (VII) Final approval of manuscript: All authors. \\ \#These authors contributed equally to this work. \\ Correspondence to: Kazuma Nagata, MD. Department of Respiratory Medicine, Kobe City Medical Center General Hospital, 2-1-1 Minatojima- \\ minamimachi, Chuo-ku, Kobe, Hyogo, 650-0047, Japan. Email: knagata@kcho.jp.
}

Background: The 4-m gait speed test is a simple functional performance measure for older adults or patients with chronic obstructive lung disease. However, limited data exist regarding these measures for patients with interstitial lung disease. We evaluated the correlation between the 4-m gait speed and 6-min walk distance tests for interstitial lung disease patients and examined their underlying physiological factors.

Methods: The 4-m gait speed and 6-min walk tests were prospectively conducted for 51 patients with interstitial lung disease. Other measurements included health-related quality of life, modified Medical Research Council score, arterial blood gas levels, pulmonary function, muscle strength according to the skeletal muscle mass index and physical activity.

Results: Thirty-five patients were male (68.6\%). Thirty-four patients had idiopathic pulmonary fibrosis $(66.7 \%)$. There were significant correlations between the 4-m gait speeds and 6-min walk distances ( $\mathrm{r}=0.57$; $\mathrm{P}<0.001)$. Multivariate analyses showed that both the 4-m gait speed and 6-min walk distance were correlated with the modified Medical Research Council score. In addition, the 6-min walk distance was correlated with age and the percentage of the predicted diffusion capacity of carbon monoxide.

Conclusions: The 4-m gait speed test is a simple, easy to perform and reliable functional performance measure for interstitial lung disease patients.

Keywords: Interstitial lung diseases (ILD); muscle strength; rehabilitation; 4-m gait speed; 6-min walk test

Submitted Aug 18, 2019. Accepted for publication Feb 18, 2020.

doi: $10.21037 /$ jtd.2020.02.57

View this article at: http://dx.doi.org/10.21037/jtd.2020.02.57

\section{Introduction}

Interstitial lung disease (ILD) is a chronic progressive disease that is associated with increased morbidity and mortality. ILD is also associated with many symptoms, including shortness of breath and cough; as the disease progresses, these symptoms impair the activities of daily living (ADL) and health related quality of life (HRQOL) (1). 
Therefore, it is very important to use ADL to evaluate the disease progress and determine an appropriate treatment.

The 6 -min walk test $(6 M W T)$ is a practical and simple tool correlated to poor HRQOL (2), which used to determine lung function impairment $(2,3)$, physical inactivity (4) and mortality (2,5-7) in ILD patients. However, ILD causes dyspnea or exertional desaturation, which may make it difficult or impossible to perform the 6MWT. Therefore, clinical data of the 6MWT for ILD patients are limited.

Usual gait speed and 4-m gait speed (4MGS) are established surrogate markers of sarcopenia (8) and frailty (9). The 4-m gait speed test (4MGT) correlated to incremental shuttle walking test (ISW) results, HRQOL, physical activity (10) and pulmonary functional capacity (11) in chronic obstructive pulmonary disease (COPD) patients. However, little previous reports have shown the usefulness of $4 M G S$ for ILD patients.

The primary purpose of the present study was to determine the efficacy of 4MGS for ILD patients by comparing the efficacy of $6 \mathrm{MWT}$ and $4 \mathrm{MGT}$. The secondary purpose of this study was to consider the parameters that are related to $4 \mathrm{MGS}$ and $6 \mathrm{MWT}$ by investigating the correlations among parameters such as arterial blood gas levels, pulmonary function test (PFT) results, HRQOL, muscle strength according to skeletal muscle mass index (SMI) and physical activity in ILD patients.

\section{Methods}

\section{Subjects}

This study is a prospective cohort study performed at Kobe City Medical Center General Hospital from November 2017 to February 2018. Fifty-two patients with chronic fibrosing ILD who came to the outpatient yard within the observational period were eligible for the criteria and 51 patients are included (one patient did not consent to the study). All patients included in this study were older than 18 years and were diagnosed according to clinical, radiological and histopathological findings. They had been diagnosed with any ILD by the radiological finding that linear or reticulonodular interstitial infiltrates on chest computed tomography, or consistent histopathological findings.

Idiopathic pulmonary fibrosis (IPF) was diagnosed according to the 2011 American Thoracic Society/Europe Respiratory Society/Japanese Respiratory Society/Latin American Thoracic Association official statement (12). The diagnosis was determined by high-resolution computed tomography (HRCT) images and surgical lung biopsy specimens. If the lung biopsy results were not available, IPF was diagnosed based on the eligibility criteria from the guideline. Collagen vascular disease-associated ILD (CVDILD) was diagnosed from the symptom, autoimmune antibody, HRCT images and histopathological findings.

Patients were excluded if they experienced difficulty understanding or completing the questionnaire, if they had an active cardiovascular disease (e.g., poorly controlled severe heart failure or arrhythmia, cardiomyopathy, or vascular disorders), a predominantly obstructive lung disease diagnosed from the result of pulmonary function test, difficulty walking because of orthopaedic conditions, or a history of acute exacerbation of ILD within 1 month of the study inclusion.

This study was approved by the Institutional Review Board of Kobe City Medical Center General Hospital (No. zn170913). All patients were provided with written informed consent.

\section{The 4MGS and 6MWT}

Patients performed the 4MGS test and 6MWT in the same day. All patients performed in the order of 4MGS, 6MWT. If needed, the patients used adequate supplemental oxygen which they prescribed in the outpatient yard.

4MGS test performed according to standardized methods by trained operators at the rehabilitation room (10). The test was performed twice, and the faster results were recorded as the gait speed. Patients walked $4 \mathrm{~m}$ and then another $1 \mathrm{~m}$ to determine the acceleration and deceleration zones.

The 6MWT was performed once, using modified criteria. In this study, patients walked around a circular course for $6 \mathrm{~min}$; however, apart from this change, the other protocol followed the American Thoracic Society criteria (13). The following parameters were assessed: distance walked within $6 \mathrm{~min}$, baseline arterial oxygen saturation (according to pulse oximetry), minimum arterial oxygen saturation during exercise (according to pulse oximetry) and modified Borg dyspnea score (determined immediately after exercise). 


\section{HRQOL questionnaires and assessments of dyspnea and cough}

All patients completed the St. George's Respiratory Questionnaire (SGRQ) Japanese test version 1, which has been validated for use in the Japanese patient population $(14,15)$. Further, all patients completed the COPD Assessment Test (CAT), Japanese version, which has been validated for use in the Japanese patient population, and the modified Medical Research Council (mMRC) dyspnea scale (16).

\section{Pulmonary function tests and analysis of arterial blood gas}

Pulmonary function tests (PFTs) were performed by welltrained operators in accordance with the American Thoracic Society/European Respiratory Society guidelines (17) and using the CHEST- $8800^{\circledR}$ system (Chest, Tokyo, Japan).

Arterial blood gas samples were collected after breathing ambient air for $5 \mathrm{~min}$ with the patient in a sitting position. The $\mathrm{pH}, \mathrm{PaO}_{2}, \mathrm{PaCO}_{2}, \mathrm{HCO}_{3}^{-}$and base excess of the blood gas samples were evaluated.

\section{Muscle strength and skeletal muscle mass index}

The quadriceps muscle strength was used to evaluate muscle strength and was measured using a hand-held dynamometer $\mu$ TasF- $1^{\circledR}$ (anima, Tokyo, Japan) with the patient in the sitting position with the knee flexed at $90^{\circ}$ over the end of the bed. Forced isometric knee extension movements were measured for $5 \mathrm{~s}$ twice on each side [quadriceps twitch force (TwQ), Nm]. Using the TwQ maximum value and body weight $(\mathrm{kg})$ measurements, the quadriceps maximal voluntary contraction force (QMVC; $\mathrm{Nm} / \mathrm{kg}$ ) was measured and recorded as the muscle strength.

The skeletal muscle mass index (SMI) score was measured by bioelectrical impedance analysis by InBody S10 ${ }^{\circledR}$ (Inbody Japan, Tokyo, Japan) and calculated using the appendicular SMI score and height.

\section{Physical activity}

Physical activity was measured by uniaxial accelerometry Lifecorder GS ${ }^{\circledR}$ (SUZUKEN, Aichi, Japan). Participants used the equipment for 1 week to measure their daily steps. The daily steps, except for those on the first and last days, were counted and averaged (steps per day); these steps were considered as the physical activity.

\section{Statistical analysis}

Demographics are presented as numbers (\%), mean \pm standard deviation (SD), or median [interquartile range (IQR)]. Correlations between the 6-min walk distance (6MWD) and 4MGS were assessed using Spearman's correlation coefficients.

Forward stepwise multiple regression models were constructed to identify the variables that best predicted the $6 \mathrm{MWD}$ and $4 \mathrm{MGS}$. Independent variables included age, percentage of predicted vital capacity, percentage of predicted diffusing capacity of carbon monoxide, $\mathrm{PaO}_{2}$, 6MWD, mMRC dyspnea score and quadriceps muscle strength. Dependent variables were the 6MWD and 4MGS. Statistical analyses were performed using JMP version 8.0 software (SAS Institute Inc., Cary, NC, USA) for Windows. Two-tailed $\mathrm{P}$ values $<0.05$ were considered significant.

\section{Results}

\section{Patient characteristics}

Table 1 shows the baseline patient characteristics of the patients participating in the study. The study included 51 patients [mean age, $74.2 \pm 7.5$ years; 35 males $(69 \%)$ ]. Seventeen patients (33\%) were non-smokers, $29(57 \%)$ were ex-smokers (former smokers) and $5(10 \%)$ were current smokers. Six patients (12\%) needed supplemental oxygen.

Thirty-four participants diagnosed as IPF (67\%), 13 had collagen-vascular disease-associated ILD (25\%) and 4 had other ILDs (8\%). Collagen-vascular disease-associated ILD included dermatomyositis-associated ILD ( $\mathrm{n}=7 ; 13 \%)$, rheumatoid arthritis-associated ILD ( $\mathrm{n}=2 ; 4 \%)$, antineutrophil cytoplasmic antibody-associated ILD ( $\mathrm{n}=2 ; 4 \%)$, systemic sclerosis-related ILD ( $\mathrm{n}=1 ; 2 \%)$ and Sjögren's syndrome-associated ILD ( $\mathrm{n}=1 ; 2 \%)$. The category "other ILDs" (biopsy not performed, non-IPF) included ILD patients who did not undergo biopsy but were suspected of having idiopathic non-specific IP, chronic hypersensitivity pneumonia, or smoking-related ILD based on clinical and radiological findings.

Table 2 shows lung function measurements, arterial blood gas measurements, physical capacity and physical activity. There was a loss of data about physical activity in only one patient. The pulmonary function test showed normal-to-severe volume capacity $(2.58 \pm 0.74 \mathrm{~L})$, percentage of predicted volume capacity $(82.1 \% \pm 15.9 \%)$ and percentage of predicted diffusion capacity of carbon 
Table 1 Baseline characteristics of study participants

\begin{tabular}{lc}
\hline Characteristics & Values \\
\hline Male & $35(69 \%)$ \\
Age, years & $74.2 \pm 7.5$ \\
Height, cm & $161.1 \pm 9.1$ \\
Weight, kg & $62.0 \pm 12.9$ \\
BMI, kg/m ${ }^{2}$ & $23.7 \pm 3.5$ \\
Smoking status & \\
Non/ex/current & $17(33 \%) /$ \\
& $29(57 \%) / 5(10 \%)$ \\
Diagnosis & \\
IPF & $34(67 \%)$ \\
CVD-associated ILD & $13(25 \%)$ \\
DM-associated ILD & $7(13 \%)$ \\
RA-associated ILD & $2(4 \%)$ \\
ANCA-associated ILD & $2(4 \%)$ \\
SSc-associated ILD & $1(2 \%)$ \\
SjS-associated ILD & $1(2 \%)$ \\
Other (biopsy not performed, non-IPF) & $4(8 \%)$ \\
Use of supplemental oxygen & $6(12 \%)$ \\
\hline
\end{tabular}

Non, non-smoker; ex, former smoker; BMI, body mass index; IPF, idiopathic pulmonary fibrosis; CVD, collagen-vascular disease; DM; dermatomyositis RA, rheumatoid arthritis; ANCA, anti-neutrophil cytoplasmic antibody; SSc, systemic sclerosis; SjS, Sjögren's syndrome; ILD, interstitial lung disease.

monoxide $(73.8 \% \pm 26.4 \%)$. The arterial blood gas analysis suggested normal-to-severe $\mathrm{PaO}_{2}(83.5 \pm 21.6 \mathrm{mmHg})$, but the blood gas $\mathrm{pH}$ was completely normal $(7.40 \pm 0.03)$. Physical capacity was measured using quadriceps femoris torque $(1.57 \pm 0.53 \mathrm{Nm} / \mathrm{kg})$, grip strength $(28.0 \pm 8.5 \mathrm{~kg})$, physical activity $(3,709.6 \pm 725.9$ steps/day) and SMI $\left(7.32937 \pm 1.26356 \mathrm{~kg} / \mathrm{m}^{2}\right)$. Physical activity was not evaluated for one patient because that patient stopped attending follow-up examinations and the device could not be recovered. The walking distance, minimal $\mathrm{SpO}_{2}$ and Borg scale score after the $6 \mathrm{MWT}$ were $429.1 \pm 123.9 \mathrm{~m}$, $88.7 \% \pm 5.8 \%$ and 4 (range, $2-5$ ), respectively. The $4 \mathrm{MGS}$ varied widely between individuals with severe impairment to normal $(1.05 \pm 0.24 \mathrm{~m} / \mathrm{s})$.

The mMRC dyspnea scores, scores for each domain of SGRQ [median, 31.8 (IQR, 21.2-55.1) for SGRQ-symptom score; median, 47.46 (IQR, 29.33-66.19) for SGRQ-action score; and median, 17.82 (IQR, 5.70-35.21) for SGRQimpact score], total SGRQ score [30.64 (17.421-43.130)] and CAT scores $(11 ; 5-19)$ are summarised in Table 3. These scores varied from severe impairment to normal status, but $62.7 \%$ of participants had an mMRC score of 0 or 1 .

Figure 1 shows the correlation between 4MGS and $6 \mathrm{MWD}$. There was a significant correlation between 4 MGS and 6MWD $(\mathrm{r}=0.57 ; \mathrm{P}<0.0001)$. Table 4 shows the relationships and differences between the $4 \mathrm{MGS}$ or $6 \mathrm{MWD}$ and other outcome measures for this cohort. The 4MGS was significantly correlated with the mMRC score, CAT score, SGRQ total score and each domain of the SGRQ score (symptom score, activity score and impact score). The $6 \mathrm{MWD}$ was significantly correlated with patient age, $\mathrm{PaO}_{2}$, percentage of diffusion capacity of carbon monoxide, mMRC score, CAT score, SGRQ total score and each domain of the SGRQ score.

Table 5 shows the results of the forward stepwise multiple regression analyses that identified the variables that were correlated with $4 \mathrm{MGS}$ and $6 \mathrm{MWD}$. The $6 \mathrm{MWD}$ was correlated with patient age, $\mathrm{PaO}_{2}$, \% DLCO, mMRC score and SMI score. However, the 4MGS was associated only with the mMRC score.

\section{Discussion}

This study showed a significant correlation between the 4MGS and 6MWD for ILD patients. It also showed that dyspnea was correlated with both the 4MGS and 6MWD, whereas the pulmonary function and SMI scores were not correlated with the $4 \mathrm{MGS}$.

In general, the $6 \mathrm{MWT}$ is the most widely used and reliable tool for ILD patients. However, this test had several limitations. First, the test requires a wide $30-\mathrm{m}$ course. Second, dyspnea and desaturation for ILD patients occur during exercise, limiting the walking distance; thus, this walking distance may not be appropriate for evaluating all patients. The 4MGS does not have established utility, but it does have several attractive properties. It is inexpensive, can be performed quickly and requires little space (18). Because most adults are familiar with walking, the test is easy to understand and has been shown to be reliable, even when used for individuals with cognitive dysfunction.

The 4MGS has been recommended as a potentially useful marker of multisystemic well-being for communitydwelling older adults (19). Slow 4MGS is a consistent risk factor for disability, cognitive impairment, nursing home admission, falls, cardiovascular mortality and all- 
Table 2 Lung function and functional capacity of the participants

\begin{tabular}{|c|c|c|}
\hline & Mean \pm SD & Range \\
\hline FVC, L & $2.57 \pm 0.74$ & $1.24-4.19$ \\
\hline VC, predicted \% & $82.1 \pm 15.9$ & $49-117.2$ \\
\hline $\mathrm{FEV}_{1}, \mathrm{~L}$ & $2.06 \pm 0.57$ & $0.98-3.3$ \\
\hline $\mathrm{FEV}_{1} / \mathrm{FVC}$ & $81.3 \pm 6.4$ & $68.3-95.9$ \\
\hline DLCO, predicted \% & $73.8 \pm 26.4$ & $21.1-133.3$ \\
\hline $\mathrm{PaO} 2, \mathrm{mmHg}$ & $83.5 \pm 21.6$ & $37.1-173$ \\
\hline $\mathrm{PaCO} 2, \mathrm{mmHg}$ & $39.0 \pm 3.7$ & $32.9-52.9$ \\
\hline QF torque, $\mathrm{Nm} / \mathrm{kg}$ & $1.57 \pm 0.53$ & $0.67-3.59$ \\
\hline Grip strength, kg & $28.0 \pm 8.5$ & $14.5-49.1$ \\
\hline SMI, $\mathrm{kg} / \mathrm{m}^{2}$ & $7.32937 \pm 1.26356$ & $4.70539-10.4145$ \\
\hline \multicolumn{3}{|l|}{ Six-minute walk test } \\
\hline Walk distance, $\mathrm{m}$ & $429.1 \pm 123.9$ & $63.0-679.0$ \\
\hline Minimal $\mathrm{SpO}_{2}, \%$ & $88.7 \pm 5.8$ & $72.0-96.0$ \\
\hline Borg scale score & $4[2-5]$ & $0-10$ \\
\hline Four-meter gait speed, m/s & $1.05 \pm 0.24$ & $0.55-1.71$ \\
\hline
\end{tabular}

SD, standard deviation; VC, volume capacity; FVC, forced volume capacity; FEV $_{1}$, forced expiratory volume in 1 s; DLCO, diffusing capacity for carbon monoxide; $\mathrm{PaO}_{2}$, arterial oxygen pressure; $\mathrm{PaCO}_{2}$, arterial carbon dioxide; QF, quadriceps force; SMI, skeletal muscle mass index; $\mathrm{SpO}_{2}$, percutaneous arterial oxygen saturation.

Table 3 Health status and HRQOL scores

\begin{tabular}{lcc}
\hline & Result & Range \\
\hline MRC score (0/1/2/3/4) & $19 / 13 / 10 / 6 / 3$ & $0-4$ \\
CAT & $11[5-19]$ & $0-40$ \\
SGRQ total score & $30.64(17.421-43.130)$ & $0-88.49$ \\
SGRQ-S (IQR) & $31.8(21.2-55.1)$ & $0-90.6$ \\
SGRQ-A (IQR) & $47.46(29.33-66.19)$ & $0-93.42$ \\
SGRQ-I (IQR) & $17.82(5.70-35.21)$ & $0-90.88$ \\
\hline
\end{tabular}

CAT, chronic obstructive pulmonary disease assessment test; MRC, Medical Research Council; HRQOL, health-related quality of life; $S G R Q$, St. George's respiratory questionnaire.

cause mortality (19-22). The 4MGS is an established marker of the exercise capacity of the elderly, and it has been correlated with other function measurements $(23,24)$.
The 4MGS is associated with age (25), HRQOL (26), dyspnea (26), lung function (25) and functional capacity $(11,26)$ of COPD patients. We could not find previous 
studies on the correlation between muscle strength and 4MGS. However, the results of other studies cannot be compared because there is no standardised method for

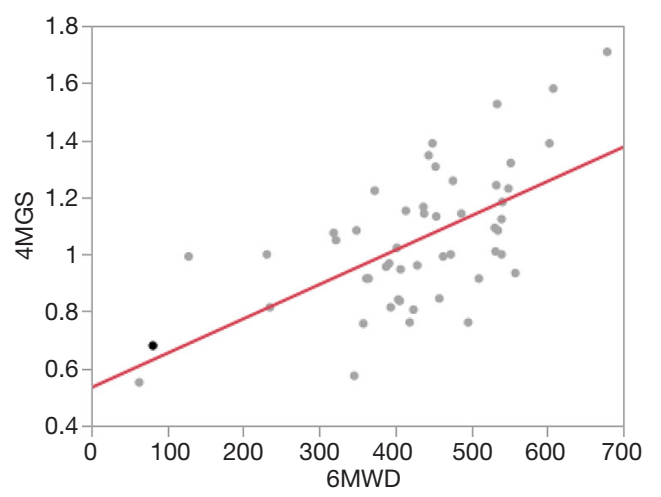

Figure 1 Correlation between 4-m gait speed and 6-m walk distance. $\mathrm{r}=0.57 ; \mathrm{P}<0.0001$. measuring the usual gait speed, including 4MGS.

There have been several reports of correlations between 6MWD and 4MGS in ILD patients. DePew et al. (27) studied 70 individuals with chronic lung disease ( $\mathrm{n}=55$ ILD; IPF data not reported) and demonstrated a strong positive correlation between the 4MGS and 6MWD $(\mathrm{r}=0.70)$ and a significant negative correlation with $\mathrm{mMRC}$ score $(\mathrm{r}=0.44)$ for the entire group. However, this study included not only ILD patients but also other respiratory failure patients. Nolan et al. (28) studied 111 patients ( $\mathrm{n}=46$ baseline; $\mathrm{n}=65$ validation) with IPF and demonstrated a significant correlation between the 4MGS and 6MWD ( $\mathrm{r}=0.76$; $\mathrm{P}<0.0001)$. However, in Nolan's study, they did not study muscle strength and physical activity, and the participants were limited to outpatients attending specialist ILD clinics at tertiary centres. Our study also showed a significant correlation between 4MGS and 6MWD, which supported the efficacy of $4 M G S$ in ILD patients. The $4 M G T$ is a

Table 4 Relationships and differences between 4MGS and 6MWD and other outcome measures in the study cohort

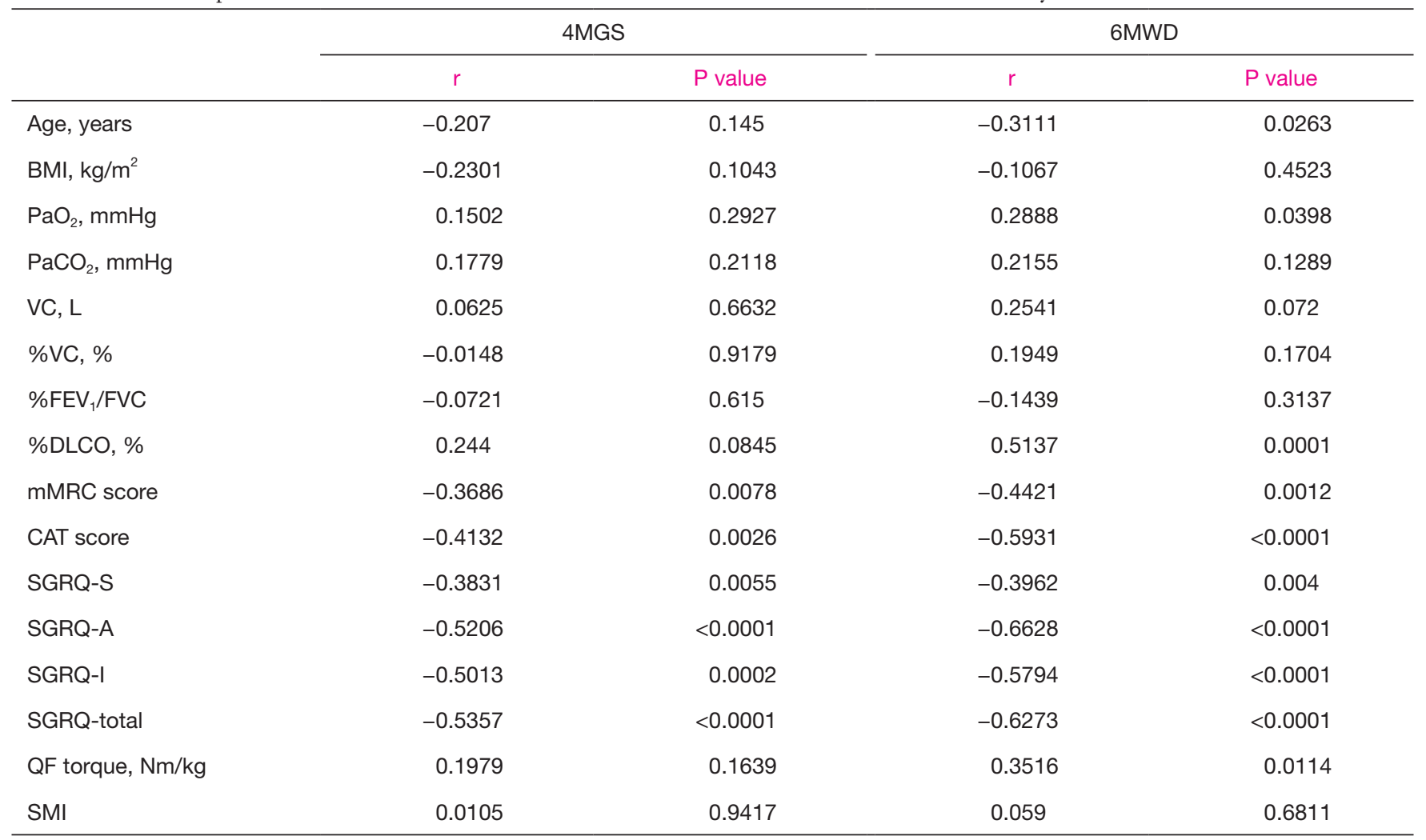

4MGS, 4-m gait speed; 6MWD, 6-min walk distance; $\mathrm{BMI}$, body mass index; $\mathrm{PaO}_{2}$, arterial oxygen pressure; $\mathrm{PaCO}_{2}$, arterial carbon dioxide pressure; VC, volume capacity; $\mathrm{FEV}_{1}$, forced expiratory volume in $1 \mathrm{~s}$; FVC, forced volume capacity; DLCO, diffusing capacity of carbon monoxide; mMRC, modified Medical Research Council; CAT, chronic obstructive pulmonary disease assessment test; SGRQ, St. George's respiratory questionnaire; QF, quadriceps force; SMI, skeletal muscle mass index. 
Table 5 Forward stepwise multiple regression models identifying the relationships between 4MGS and 6MWD and other outcome measures in the study cohort

\begin{tabular}{|c|c|c|c|c|}
\hline & \multicolumn{2}{|c|}{ 4MGS } & \multicolumn{2}{|c|}{ 6MWD } \\
\hline Age, years & -0.207 & 0.145 & -0.3111 & 0.0263 \\
\hline \%DLCO, \% & & & 0.5137 & 0.0001 \\
\hline mMRC score & -0.3686 & 0.0078 & -0.4421 & 0.0012 \\
\hline
\end{tabular}

4MGS, 4-m gait speed; 6MWD, 6-min walk distance; DLCO, diffusing capacity of carbon monoxide; mMRC, modified Medical Research Council; QF, quadriceps force.

simple functional performance measure and stratification tool that is correlated with $6 \mathrm{MWT}$ in general hospital settings.

In this study, we investigated the parameters that were related to the correlation between 4MGS and 6MWD by investigating the correlation of these measures with arterial blood gas, PFT, QOL, muscle strength, SMI and physical activity. Dyspnea score of SGRQ and mMRC scores correlated well with 4MGS. However, both univariate and multivariate analyses found no significant correlation between the $4 \mathrm{MGS}$ and age, respiratory function, $\mathrm{PaO}_{2}$, muscle strength and SMI.

For ILD patients, the $6 \mathrm{MWT}$ was correlated with symptoms (29), HRQOL (30), lung function (29) and functional capacity (30). Desaturation and 6MWD were correlated with mortality (30,31), and pulmonary rehabilitation increased the 6MWD (32). Data concerning the 4MGS for ILD patients are limited, but a previous study has shown a significant correlation between $4 \mathrm{MGS}$ and dyspnea or poor prognosis, despite the lung capacity or HRCT parameters (28).

Our data indicated that $6 \mathrm{MWD}$ correlated with more factors than 4MGS. Although 4MGS correlated with the disease symptoms, it did not correlate with age, lung capacity, muscle strength and SMI score. Previous studies have indicated that $4 \mathrm{MGS}$ is not a measure of peak exercise capacity $(26,28)$, and therefore, 4 MGS does not correlate with lung capacity, muscle strength and SMI score. Our study supported the previous data and suggested that poor 4MGS did not result from skeletal muscle weakness or decreased lung function; instead, it resulted from dyspnea.

The present study had several limitations. First, this was a single-centre study and there was selection bias in the patient population. Second, this study population was heterogeneous because various ILD patients were included without their ILD phenotype being distinguished. The histology of some patients was not evaluated because of age, impaired ADL, or refusal of the patient. Third, some of patients with relatively mild to moderate respiratory dysfunction were included in the study. Fourth, the $6 \mathrm{MWT}$ were performed only once because of patients age or impaired ADL and it may be several errors in the result of the test. The additive research about the more severe respiratory dysfunction patients will be needed.

\section{Conclusions}

The 4MGS was significantly correlated with the 6MWD. Furthermore, $4 M G S$ can be a useful evaluation tool for ILD patients, especially those who cannot perform the 6MWT. However, 4MGS has a weaker correlation with age, lung capacity and muscle strength than $6 \mathrm{MWD}$, indicating that $6 \mathrm{MWD}$ reflects more factors than 4MGS. the utility of the $4 M G S$ as a functional performance measure is uncertain. The longitudinal validity, minimal change of significance, prognostic value for 4 MGS in ILD populations are unknown.

\section{Acknowledgments}

The authors thank the Department of Respiratory Medicine and the Department of Rehabilitaion for their clinical support.

Funding: None.

\section{Footnote}

Conflicts of Interest: All authors have completed the ICMJE uniform disclosure form (available at http://dx.doi. org/10.21037/jtd.2020.02.57). KT get personal fees from 
Boeringer-Ingelheim, personal fees from Shionogi, personal fees from Teijin pharma, outside the submitted work. The other authors have no conflicts of interest to declare.

Ethical Statement: The authors are accountable for all aspects of the work in ensuring that questions related to the accuracy or integrity of any part of the work are appropriately investigated and resolved. This study was approved by the Institutional Review Board of Kobe City Medical Center General Hospital (No. zn170913). All patients were provided with written informed consent.

Open Access Statement: This is an Open Access article distributed in accordance with the Creative Commons Attribution-NonCommercial-NoDerivs 4.0 International License (CC BY-NC-ND 4.0), which permits the noncommercial replication and distribution of the article with the strict proviso that no changes or edits are made and the original work is properly cited (including links to both the formal publication through the relevant DOI and the license). See: https://creativecommons.org/licenses/by-nc-nd/4.0/.

\section{References}

1. Swigris JJ, Kuschner WG, Jacobs SS, et al. Health-related quality of life in patients with idiopathic pulmonary fibrosis: a systematic review. Thorax 2005;60:588-94.

2. Singh SJ, Puhan MA, Andrianopoulos V, et al. An official systematic review of the European Respiratory Society/

American Thoracic Society: measurement properties of field walking tests in chronic respiratory disease. Eur Respir J 2014;44:1447-78.

3. Agusti A, Calverley PM, Celli B, et al. Characterisation of COPD heterogeneity in the ECLIPSE cohort. Respir Res 2010;11:122.

4. Pitta F, Troosters T, Spruit MA, et al. Activity monitoring for assessment of physical activities in daily life in patients with chronic obstructive pulmonary disease. Arch Phys Med Rehabil 2005;86:1979-85.

5. Pinto-Plata VM, Cote C, Cabral H, et al. The 6-min walk distance: change over time and value as a predictor of survival in severe COPD. Eur Respir J 2004;23:28-33.

6. Cote CG, Casanova C, Marin JM, et al. Validation and comparison of reference equations for the 6-min walk distance test. Eur Respir J 2008;31:571-8.

7. Polkey MI, Spruit MA, Edwards LD, et al. Sixminute-walk test in chronic obstructive pulmonary disease: minimal clinically important difference for death or hospitalization. Am J Respir Crit Care Med 2013;187:382-6.

8. Cruz-Jentoft AJ, Baeyens JP, Bauer JM, et al. Sarcopenia: European consensus on definition and diagnosis: Report of the European Working Group on Sarcopenia in Older People. Age Ageing 2010;39:412-23.

9. Fried LP, Tangen CM, Walston J, et al. Frailty in older adults: evidence for a phenotype. J Gerontol A Biol Sci Med Sci 2001;56:M146-56.

10. Kon SS, Patel MS, Canavan JL, et al. Reliability and validity of 4-metre gait speed in COPD. Eur Respir J 2013;42:333-40.

11. Ilgin D, Ozalevli S, Kilinc O, et al. Gait speed as a functional capacity indicator in patients with chronic obstructive pulmonary disease. Ann Thorac Med 2011;6:141-6.

12. Raghu G, Collard HR, Egan JJ, et al. An official ATS/ ERS/JRS/ALAT statement: idiopathic pulmonary fibrosis: evidence-based guidelines for diagnosis and management. Am J Respir Crit Care Med 2011;183:788-824.

13. ATS Committee on Proficiency Standards for Clinical Pulmonary Function Laboratories. ATS statement: guidelines for the six-minute walk test. Am J Respir Crit Care Med 2002;166:111-7.

14. Jones PW, Quirk FH, Baveystock CM, et al. A selfcomplete measure of health status for chronic airflow limitation. The St. George's Respiratory Questionnaire. Am Rev Respir Dis 1992;145:1321-7.

15. Hajiro T, Nishimura K, Tsukino M, et al. Comparison of discriminative properties among disease-specific questionnaires for measuring health-related quality of life in patients with chronic obstructive pulmonary disease. Am J Respir Crit Care Med 1998;157:785-90.

16. Tsuda T, Suematsu R, Kamohara K, et al. Development of the Japanese version of the COPD Assessment Test. Respir Investig 2012;50:34-9.

17. Pellegrino R, Viegi G, Brusasco V, et al. Interpretative strategies for lung function tests. Eur Respir J 2005;26:948-68.

18. Karpman C, Lebrasseur NK, Depew ZS, et al. Measuring gait speed in the out-patient clinic: methodology and feasibility. Respir Care 2014;59:531-7.

19. Abellan van Kan G, Rolland Y, Andrieu S, et al. Gait speed at usual pace as a predictor of adverse outcomes in community-dwelling older people an International Academy on Nutrition and Aging (IANA) Task Force. J Nutr Health Aging 2009;13:881-9.

20. Dumurgier J, Elbaz A, Ducimetiere P, et al. Slow walking 
speed and cardiovascular death in well functioning older adults: prospective cohort study. BMJ 2009;339:b4460.

21. Guralnik JM, Ferrucci L, Simonsick EM, et al. Lowerextremity function in persons over the age of 70 years as a predictor of subsequent disability. N Engl J Med 1995;332:556-61.

22. Studenski S, Perera S, Patel K, et al. Gait speed and survival in older adults. JAMA 2011;305:50-8.

23. Buchner DM, Larson EB, Wagner EH, et al. Evidence for a non-linear relationship between leg strength and gait speed. Age Ageing 1996;25:386-91.

24. Steffen TM, Hacker TA, Mollinger L. Age- and genderrelated test performance in community-dwelling elderly people: Six-Minute Walk Test, Berg Balance Scale, Timed Up \& Go Test, and gait speeds. Phys Ther 2002;82:128-37.

25. Bernabeu-Mora R, Medina-Mirapeix F, LlamazaresHerran E, et al. The accuracy with which the 5 times sitto-stand test, versus gait speed, can identify poor exercise tolerance in patients with COPD: A cross-sectional study. Medicine (Baltimore) 2016;95:e4740.

26. Kon SS, Jones SE, Schofield SJ, et al. Gait speed and readmission following hospitalisation for acute exacerbations of COPD: a prospective study. Thorax

Cite this article as: Hirabayashi R, Takahashi Y, Nagata K, Morimoto T, Wakata K, Nakagawa A, Tachikawa R, Otsuka K, Tomii K. The validity and reliability of four-meter gait speed test for stable interstitial lung disease patients: the prospective study. J Thorac Dis 2020;12(4):1296-1304. doi: 10.21037/ jtd.2020.02.57
2015;70:1131-7.

27. DePew ZS, Karpman C, Novotny PJ, et al. Correlations between gait speed, 6-minute walk distance, physical activity, and self-efficacy in patients with severe chronic lung disease. Respir Care 2013;58:2113-9.

28. Nolan CM, Maddocks M, Maher TM, et al. Phenotypic characteristics associated with slow gait speed in idiopathic pulmonary fibrosis. Respirology 2018;23:498-506.

29. Nathan SD, du Bois RM, Albera C, et al. Validation of test performance characteristics and minimal clinically important difference of the 6-minute walk test in patients with idiopathic pulmonary fibrosis. Respir Med 2015;109:914-22.

30. du Bois RM, Weycker D, Albera C, et al. Six-minute-walk test in idiopathic pulmonary fibrosis: test validation and minimal clinically important difference. Am J Respir Crit Care Med 2011;183:1231-7.

31. Flaherty KR, Andrei AC, Murray S, et al. Idiopathic pulmonary fibrosis: prognostic value of changes in physiology and six-minute-walk test. Am J Respir Crit Care Med 2006;174:803-9.

32. Dowman LM, McDonald CF, Hill CJ, et al. The evidence of benefits of exercise training in interstitial lung disease: a randomised controlled trial. Thorax 2017;72:610-9. 